\title{
THE DOMINATED ERGODIC ESTIMATE FOR MEAN BOUNDED, INVERTIBLE, POSITIVE OPERATORS
}

\author{
F. J. MARTIN-REYES AND A. DE LA TORRE
}

(Communicated by John B. Conway)

\begin{abstract}
We characterize those positive linear operators with positive inverse for which the dominated ergodic estimate holds. We also prove that for such operators one has mean and a.e. convergence.
\end{abstract}

1. Introduction. Akcoglu proved [1] a Dominated Ergodic Theorem for positive contractions in $L_{p}, 1<p<\infty$. It seems natural to try to extend this result to power bounded operators, but this is still an open problem. Brunel and Emilion [3] have shown that a positive answer to the above problem would imply an ergodic theorem for operators having uniformly bounded averages.

Recently, the authors proved [8] that if $T$ is an invertible positive linear operator with positive inverse on $L_{p}, 1<p<\infty$, of a $\sigma$-finite measure space then $\sup _{n \geq 0}\left\|(2 n+1)^{-1} \sum_{i=-n}^{n} T^{i}\right\|_{p}<\infty$ implies that a dominated ergodic theorem holds for the maximal operator $\sup _{n \geq 0}(2 n+1)^{-1} \sum_{i=-n}^{n}\left|T^{i} f\right|$. In this note we see that the same holds for the averages $(n+1)^{-1} \sum_{i=0}^{n} T^{i}$ and the corresponding maximal operator. Therefore our result generalizes Akcoglu's theorem to mean bounded operators when $T$ and $T^{-1}$ are positive. As a consequence, we get a.e. convergence of the averages. Since there are examples of Feder [6] and Assani [2] which show that this is false if the positivity is dropped, our result closes the case of invertible operators, and seems to indicate that the corresponding theorem for power bounded operators (invertible or not) might be true.

Throughout this paper $T$ will denote a positive linear operator with positive inverse on $L_{p}, 1<p<\infty, C$ will mean a positive constant not necessarily the same at each occurrence, and $\mathbf{N}$ and $\mathbf{Z}$ will stand for the set of natural integers and the set of integers respectively.

2. The dominated estimate. Let $(X, \mathscr{F}, \mu)$ be a $\sigma$-finite measure space and let $T$ be an invertible positive linear operator on $L_{p}=L_{p}(X, \mathscr{F}, \mu)$, with $1<p<$ $\infty$, and suppose $T^{-1}$ is also positive. Then, as is well known [7], $T$ and $T^{-1}$ are Lamperti operators and they have the following properties:

(a) For each integer $i$, there exists a positive function $g_{i}$ such that

$$
T^{i} f=g_{i} S^{i} f \text { and } g_{i+j}=g_{i} S^{i} g_{j}
$$

where $S$ is a positive and multiplicative invertible linear map acting on measurable functions.

Received by the editors January 1, 1987.

1980 Mathematics Subject Classification (1985 Revision). Primary 47A35; Secondary 46E30.

Key words and phrases. Dominated Ergodic Theorem, Individual Ergodic Theorem, positive invertible operators.

This research has been supported by C.A.I.C.Y.T. Grant (PB85-0434). 
(b) For each integer $i$, there exists a positive function $J_{i}$ such that

$$
J_{i+j}=J_{i} S^{i} J_{j} \text { and } \int_{X} J_{i} S^{i} f d \mu=\int_{X} f d \mu .
$$

(c) For each integer $i$, the adjoint of $T^{i}$ is the operator

$$
\left(T^{i}\right)^{*} f=\left(g_{-i}\right)^{-1} J_{-i} S^{-i} f \text {. }
$$

From these properties we have the following:

(d) If $h_{i}=g_{i}^{-p} J_{i}$ then $\int_{X}\left|T^{i} f\right|^{p} h_{i} d \mu=\int_{X}|f|^{p} d \mu$. in $L_{q}$

(e) Let $q=\left(1-p^{-1}\right)^{-1}$ be the conjugate exponent of $p$. Then for every $f \geq 0$

$$
h_{i}\left(T^{i} f^{q-1}\right)^{p-1}=\left(T^{-i}\right)^{*} f .
$$

For every pair of naturals, $k$ and $n$, we will consider the average $T_{k, n}$ defined by

$$
T_{k, n} f=(n+k+1)^{-1} \sum_{i=-k}^{n} T^{i} f
$$

and the maximal operator $M^{+}$defined by

$$
M^{+} f=\sup _{n \geq 0} T_{0, n}|f| \text {. }
$$

Our main theorem establishes that $\sup _{n \geq 0}\left\|T_{0, n}\right\|_{p}<\infty$ implies that a dominated ergodic theorem holds for the maximal operator $M^{+}$. The proof will need the following result about weights for the one-sided Hardy-Littlewood maximal function in $\mathbf{Z}$ (see [9]).

LEMMA. If $f$ is a function on $\mathbf{Z}$, let $f^{*}(j)=\sup _{n \geq 0}(n+1)^{-1} \sum_{i=0}^{n}|f(i+j)|$. Let $w$ be a positive function on $\mathbf{Z}$. There exists a constant $C>0$ such that for any $f$

$$
\sum_{i=-\infty}^{\infty}\left|f^{*}(i)\right|^{p} w(i) \leq C \sum_{i=-\infty}^{\infty}|f(i)|^{p} w(i)
$$

if and only if $w$ satisfies the following condition: $k$

$A_{p}^{+}$: There exists a constant $C>0$ such that for any integer $j$ and any natural

$$
\sum_{i=0}^{k} w(i+j)\left(\sum_{i=k}^{2 k} w^{1-q}(i+j)\right)^{p-1} \leq C(k+1)^{p}
$$

REMARK. Observe that the $A_{p}^{+}$condition is equivalent to saying that there exists a constant $C>0$ such that for any $j, r$ and $k$, with $0 \leq r \leq k$,

$$
\sum_{i=0}^{r} w(i+j)\left(\sum_{i=r}^{k} w^{1-q}(i+j)\right)^{p-1} \leq C(k+1)^{p}
$$

The constants in (1), (2) and (3) are functions of $p$ and of each other alone.

We will say that a positive function $w$ on $\mathbf{N}$ satisfies $A_{p}^{+}(\mathbf{N})$ if there exists a constant $C>0$ such that (3) holds for any $j, r$ and $k$ with $0 \leq r \leq k$ and $j \geq 0$. It can be proved that $A_{p}^{+}(\mathrm{N})$ characterizes the good weights for the maximal operator defined on functions on $\mathbf{N}$ (observe that in this case we cannot take $r$ "the middle point" between 0 and $k$ ).

Under the above assumptions and notations we have the following theorem. 
(2.1) THEOREM. The following are equivalent.

(i) There exists a constant $C>0$ such that for any $f$ in $L_{p}$

$$
\int_{X}\left|M^{+} f\right|^{p} d \mu \leq C \int_{X}|f|^{p} d \mu .
$$

(ii) There exists a constant $C>0$ such that for any $f$ in $L_{p}$

$$
\sup _{n \geq 0} \int_{X}\left|T_{0, n} f\right|^{p} d \mu \leq C \int_{X}|f|^{p} d \mu .
$$

(iii) For almost all $x$, the function defined on $\mathbf{Z}$ by $i \rightarrow h_{i}(x)$ satisfies $A_{p}^{+}$with a constant independent of $x$.

(iv) For almost all $x$, the function defined on $\mathbf{N}$ by $i \rightarrow h_{i}(x)$ satisfies $A_{p}^{+}(\mathbf{N})$ with a constant independent of $x$.

PROOF. It is clear that (ii) follows from (i).

(iii) implies (i). Assume that $f$ is nonnegative and consider for each $N>0$ the truncated maximal operator $M_{N}^{+} f(x)=\sup _{n \leq N} T_{0, n}|f|(x)$. By property (d) we get, if $L$ is a natural number,

$$
\int_{X}\left|M_{N}^{+} f\right|^{p} d \mu=\int_{X}(L+1)^{-1} \sum_{i=0}^{L}\left[T^{i}\left(M_{N}^{+} f\right)\right]^{p} h_{i} d \mu .
$$

It is clear that there exist pairwise disjoint sets $A_{0}, \ldots, A_{N}$ such that $M_{N}^{+}(f(x)=$ $\sum_{j=0}^{N} \chi_{A_{j}}(x) T_{0, j} f(x)$. Then $T^{i}\left(M_{N}^{+} f\right)(x)=\sum_{j=0}^{N} T^{i}\left(\chi_{A_{j}} T_{0, j} f\right)(x)$. Since $T^{i}$ is positive and separates supports

$$
T^{i}\left(\chi_{A_{j}} T_{0, j} f\right)(x) \leq \chi_{E_{i j}}(x) T^{i}\left(T_{0, j} f\right)(x) \leq \chi_{E_{i j}}(x) M_{N}^{+}\left(T^{i} f\right)(x)
$$

for suitable pairwise disjoint $E_{i 1}, \ldots, E_{i N}$. Therefore

$$
T^{i}\left(M_{N}^{+} f\right) \leq M_{N}^{+}\left(T^{i} f\right) \text {. }
$$

On the other hand, for every $i$ with $0 \leq i \leq L$

$$
M_{N}^{+}\left(T^{i} f\right)(x) \leq\left(H_{x}\right)^{*}(i)
$$

where $H_{x}$ is the function on $\mathbf{Z}$ defined by $H_{x}(i)=T^{i} f(x) \chi_{[0, N+L]}(i)$ and $\chi_{[0, N+L]}$ is the characteristic function of the interval $[0, N+L]$ on $\mathbf{Z}$. Then, by $(4),(5)$ and (6),

$$
\int_{X}\left|M_{N}^{+} f\right|^{p} d \mu \leq \int_{X}(L+1)^{-1} \sum_{i=-\infty}^{\infty}\left[\left(H_{x}\right)^{*}(i)\right]^{p} h_{i}(x) d \mu .
$$

Now, by (iii) and the lemma

$$
\sum_{i=-\infty}^{\infty}\left[\left(H_{x}\right)^{*}(i)\right]^{p} h_{i}(x) \leq C \sum_{i=0}^{N+L}\left[T^{i} f(x)\right]^{p} h_{i}(x) .
$$

Then, this inequality, (7) and property (d) give

$$
\int_{X}\left|M_{N}^{+} f\right|^{p} d \mu \leq C(N+L+1)(L+1)^{-1} \int_{X} f^{p} d \mu .
$$


Letting $L$ and then $N$ tend to infinity we get (i).

(ii) implies (iii). Let $j$ and $k$ be integers with $0 \leq k$. By duality we have

$$
\begin{aligned}
& \int_{X}\left|T_{0,2 k} f\right|^{p} d \mu \leq C \int_{X}|f|^{p} d \mu . \\
& \int_{X}\left|T_{0,2 k}^{*} f\right|^{q} d \mu \leq C \int_{X}|f|^{q} d \mu .
\end{aligned}
$$

Assume $p \leq 2$. By (8) and (9) we have that the operators $P: f \rightarrow\left[T_{0,2 k}|f|^{q-1}\right]^{p-1}$ and $Q: f \rightarrow T_{0,2 k}^{*} f$ are bounded in $L_{q}$. Clearly $Q$ is subadditive and so is $P$ for $p \leq 2$. Let $w_{0}$ be any positive function in $L_{q}$. Define for $i \geq 1$,

$$
w_{i}=(2 C)^{-1}(P+Q)\left(w_{i-1}\right) .
$$

Since the $L_{q}$-norm of $w_{i}$ is bounded by the norm of $w_{0}$ it is clear that the series $\sum_{i=0}^{\infty} 2^{-i} w_{i}$ defines a positive function $w$ in $L_{q}$. From the definition of $w$ follows that $P w \leq C w$ and $Q w \leq C w$ which can be written

$$
T_{0,2 k} w^{q-1} \leq C w^{q-1} \text { and } T_{0,2 k}^{*} w \leq C w .
$$

If $p>2$, one just changes the roles of $p$ and $q$. In any case we get a positive function $w$ such that (10) holds.

Now, if $j \leq i \leq j+k$ the first inequality of (10) and the positivity of $T^{i}$ give

$$
(k+1)^{-1} \sum_{s=j+k}^{j+2 k} T^{s} w^{q-1} \leq C T^{i} w^{q-1} \quad \text { a.e. }
$$

Raising to $1-p$, multiplying by $\left(T^{-i}\right)^{*} w$ and summing over $i$ we get

$$
\begin{array}{rl}
\sum_{i=j}^{j+k}\left(T^{-i}\right)^{*} & w\left(T^{i} w^{q-1}\right)^{1-p} \\
\leq C\left((k+1)^{-1} \sum_{s=j+k}^{j+2 k} T^{s} w^{q-1}\right)^{1-p} \sum_{i=j}^{j+k}\left(T^{-i}\right)^{*} w \text { a.e. }
\end{array}
$$

On the other hand, if $j+k \leq i \leq j+2 k$ the second inequality of (10) and the positivity of $\left(T^{-i}\right)^{*}$ give

$$
(k+1)^{-1} \sum_{s=j}^{j+k}\left(T^{-s}\right)^{*} w \leq C\left(T^{-i}\right)^{*} w \quad \text { a.e. }
$$

Raising to $1-q$, multiplying by $T^{i} w^{q-1}$ and summing over $i$ we get

$$
\begin{aligned}
& \sum_{i=j+k}^{j+2 k}\left(\left(T^{-i}\right)^{*} w\right)^{1-q} T^{i} w^{q-1} \\
& \quad \leq C\left((k+1)^{-1} \sum_{s=j}^{j+k}\left(T^{-s}\right)^{*} w\right)^{1-q} \sum_{i=j+k}^{j+2 k} T^{i} w^{q-1} \text { a.e. }
\end{aligned}
$$


If we raise both sides of $(12)$ to the $(p-1)$ st power, put it together with $(11)$ and keep in mind property (e), we have

$$
\sum_{i=j}^{j+k} h_{i}(x)\left[\sum_{i=j+k}^{j+2 k} h_{i}^{1-q}(x)\right]^{p-1} \leq C(k+1)^{p} \quad \text { a.e. }
$$

Since $C$ is independent of $x$ we have that (iii) is satisfied.

It is clear that (iii) implies (iv). On the other hand, (i) follows from (iv) in the same way that (i) follows from (iii) but considering $H_{x}$ defined on $\mathbf{N}$.

As a consequence of Theorem (2.1) we can get the ergodic theorem proved in [8] for the maximal operator

$$
M f=\sup _{n \geq 0} T_{n, n}|f| .
$$

In order to state and prove the theorem we will need the following definitions

(2.2) Definition. A positive function $w$ on $\mathbf{Z}$ satisfies $A_{p}, 1<p<\infty$, if there exists a constant $C>0$ such that for any integer $j$ and any natural $k$

$$
\sum_{i=0}^{k} w(i+j)\left[\sum_{i=0}^{k} w^{1-q}(i+j)\right]^{p-1} \leq C(k+1)^{p} .
$$

(2.3) Definition. A positive function $w$ on $\mathbf{Z}$ satisfies $A_{p}^{-}, 1<p<\infty$, if there exists a constant $C>0$ such that for any integer $j$ and any natural $k$

$$
\sum_{i=0}^{k} w(j-i)\left[\sum_{i=k}^{2 k} w^{1-q}(j-i)\right]^{p-1} \leq C(k+1)^{p}
$$

(2.4) THEOREM. The following are equivalent.

(i) There exists a constant $C>0$ such that for any $f$ in $L_{p}$

$$
\int_{X}|M f|^{p} d \mu \leq C \int_{X}|f|^{p} d \mu .
$$

(ii) There exists a constant $C>0$ such that for any $f$ in $L_{p}$

$$
\sup _{n \geq 0} \int_{X}\left|T_{n, n} f\right|^{p} d \mu \leq C \int_{X}|f|^{p} d \mu .
$$

(iii) For almost all $x$, the function defined on the integers by $i \rightarrow h_{i}(x)$ satisfies $A_{p}$ with a constant independent of $x$.

The theorem follows directly from Theorem (2.1) applied to $T$ and $T^{-1}$ and from the following lemma.

(2.5) LemMa. Let $1<p<\infty$. A positive function $w$ on $\mathbf{Z}$ satisfies $A_{p}$ if and only if satisfies $A_{p}^{+}$and $A_{p}^{-}$. Furthermore, if $C$ is a constant for $A_{p}^{+}$and $A_{p}^{-}$then $C_{p} C$ is a constant for $A_{p}$ where $C_{p}$ depends only on $p$.

PROOF OF THE LEMMA. It is clear that $A_{p}$ implies $A_{p}^{+}$and $A_{p}^{-}$. Conversely, for fixed $j$ and $k \geq 0$ we take the smallest natural number $r$ such that

$$
\sum_{i=0}^{k} w^{1-q}(i+j) \leq 2 \sum_{i=0}^{r} w^{1-q}(i+j)
$$


Then

$$
\sum_{i=0}^{k} w^{1-q}(i+j) \leq 2 \sum_{i=r}^{k} w^{1-q}(i+j)
$$

and therefore

$$
\begin{aligned}
\sum_{i=0}^{k} w(i+j) & {\left[\sum_{i=0}^{k} w^{1-q}(i+j)\right]^{p-1} } \\
\leq & 2^{p-1} \sum_{i=0}^{r} w(i+j)\left[\sum_{i=r}^{k} w^{1-q}(i+j)\right]^{p-1} \\
& +2^{p-1} \sum_{i=r}^{k} w(i+j)\left[\sum_{i=0}^{r} w^{1-q}(i+j)\right]^{p-1} \\
\leq & C(k+1)^{p}
\end{aligned}
$$

since $w$ satisfies $A_{p}^{+}$and $A_{p}^{-}$. The statement about the constants follows easily from the proof.

3. Almost everywhere and mean convergence. As usual, the dominated estimate allows to get results about mean and a.e. convergence

(3.1) THEOREM. (a) The averages $T_{0, n}$ are uniformly bounded in $L_{p}, 1<p<$ $\infty$, if and only if for every $f$ in $L_{p}$ the averages $T_{0, n} f$ converge in the $L_{p}$-norm.

(b) If the averages $T_{0, n}$ are uniformly bounded in $L_{p}, 1<p<\infty$, then for every $f$ in $L_{p}$ the averages $T_{0, n} f$ converge a.e.

PROOF. (a) By [4, Corollary VIII.5.4] it suffices to show that if the averages $T_{0, n}$ are uniformly bounded in $L_{p}, 1<p<\infty$, then $\lim _{n \rightarrow \infty}\left\|n^{-1} T^{n} f\right\|_{p}=0$ for characteristic functions of sets of finite measure. First, we claim that $\lim _{n \rightarrow \infty} n^{-1} T^{n} f=0$ a.e. In order to prove this, we need the following property of functions that satisfy $A_{p}^{+}$: if $w$ satisfies $A_{p}^{+}$then there exists $\varepsilon>0$, which depends only on the constant in the $A_{p}^{+}$condition, such that $w$ satisfies $A_{p-\varepsilon}^{+}$(see [9]).

Now, if the averages $T_{0, n}$ are uniformly bounded then by Theorem (2.1) the function defined on $\mathbf{Z}$ by $i \rightarrow h_{i}(x)$ satisfies $A_{p}^{+}$with a constant independent of $x$. Therefore, there is $\varepsilon>0$ such that the same functions satisfy $A_{p-\varepsilon}^{+}$with constant independent of $x$. According to Theorem (2.1), this means that the maximal operator $M^{+}\left(T_{\varepsilon}\right)$ associated with the transformation

$$
T_{\varepsilon} f(x)=\left[g_{1}(x)\right]^{r} S f(x) \quad \text { where } r=p /(p-\varepsilon)
$$

is bounded in $L_{p-\varepsilon}$. Writing

$$
n^{-1} T^{n} f(x)=n^{-1} g_{n}(x) S^{n} f(x)=n^{-\varepsilon / p}\left(g_{n}^{r}(x) S^{n} f(x) n^{-1}\right)^{1 / r}
$$

and using the fact that the second factor is dominated by $\left[M^{+}\left(T_{\varepsilon}\right) f(x)\right]^{1 / r}$, which is finite a.e., we have that $n^{-1} T^{n} f(x)$ converges to 0 a.e. On the other hand, $n^{-1} T^{n} f$ is dominated pointwise by $M^{+} f$ which is an $L_{p}$-function. Therefore, by the dominated convergence theorem we have that $n^{-1} T^{n} f$ converges to 0 in the $L_{p}$-norm. 
(b) By (a) and [4, Corollary VIII.5.2] we have that the set of functions of the type $h+f-T f$ with $h$ invariant and $f$ simple is dense in $L_{p}$. The a.e. convergence is clear for the invariant functions and it follows for the functions $f-T f$ with $f$ simple from the proof of (a). Then by Banach Principle we have that the averages $T_{0, n} f$ converge a.e. for every $f$ in $L_{p}$.

As an easy corollary we get the theorems about convergence of the averages $T_{n, n}$ (see [8]).

(3.2) COROLLARY. (a) The averages $T_{n, n}$ are uniformly bounded in $L_{p}, 1<$ $p<\infty$, if and only if for every $f$ in $L_{p}$ the averages $T_{n, n}$ converge in the $L_{p}$-norm.

(b) If the averages $T_{n, n}$ are uniformly bounded in $L_{p}, 1<p<\infty$, then for every $f$ in $L_{p}$ the averages $T_{n, n}$ converge a.e.

REMARK. Statement (a) in Theorem (3.1) is a particular case of Theorem 4.2 in [5].

\section{REFERENCES}

1. M. A. Akcoglu, A pointwise ergodic theorem in $L_{p}$-spaces, Canad. J. Math. 27 (1975), 19751982.

2. I. Assani, Sur les opérateurs a puissances bornées et le théorème ergodique ponctuel dans $L^{p}[0,1]$, $1<p<\infty$, Canad. J. Math. 38 (1986), 937-946.

3. E. Brunel and R. Emilion, Sur les opérateurs positives a moyennes bornées, C. R. Acad. Sci. Paris. I 298 (1984), 103-106.

4. N. Dunford and J. T. Schwartz, Linear operators, Part I, Interscience, New York, 1958.

5. R. Emilion, Mean-bounded operators and mean ergodic theorems, J. Funct. Anal. 61 (1985), $1-14$.

6. M. Feder, On power bounded operators and the pointwise ergodic theorem, Proc. Amer. Math. Soc. 83 (1981), 349-353.

7. C. H. Kan, Ergodic properties of Lamperti operators, Canad. J. Math. 30 (1978), 1206-1214.

8. F. J. Martin-Reyes and A. de la Torre, The dominated ergodic theorem for invertible, positive operators, Semesterbericht Funktionalanalysis Tübingen, Sommersemester 1985, pp. 143-150.

9. E. Sawyer, Weighted inequalities for the one-sided Hardy-Littlewood maximal functions, Trans. Amer. Math. Soc. 297 (1986), 53-61.

Departamento de análisis Matemático, Facultad de Ciencias, Universidad DE MÁlaga, 29071-Málaga, SPAin 